\title{
Balance of hip and trunk muscle activity is associated with increased anterior pelvic tilt during prone hip extension.
}

\section{$\operatorname{AUTHOR}(\mathrm{S})$ :}

Tateuchi, Hiroshige; Taniguchi, Masashi; Mori, Natsuko; Ichihashi, Noriaki

\section{CITATION:}

Tateuchi, Hiroshige ... [et al]. Balance of hip and trunk muscle activity is associated with increased anterior pelvic tilt during prone hip extension.. Journal of electromyography and kinesiology 2012, 22(3): 391-397

\section{ISSUE DATE:}

2012-06

URL:

http://hdl.handle.net/2433/157321

\section{RIGHT:}

(C) 2012 Elsevier Ltd.; This is not the published version. Please cite only the published version.; この論文は出版社版でありません。引用の際に は出版社版をご確認ご利用ください。 
Balance of hip and trunk muscle activity is associated with increased anterior pelvic tilt during prone hip extension

Hiroshige Tateuchi ${ }^{\mathrm{a}, *}$, Masashi Taniguchi ${ }^{\mathrm{a}}$, Natsuko Mori ${ }^{\mathrm{a}}$, Noriaki Ichihashi ${ }^{\mathrm{a}}$

${ }^{a}$ Graduate School of Medicine, Kyoto University, Kyoto, Japan

Key words:

Electromyography

Prone hip extension

Muscle balance

Pelvic tilt

* Corresponding author:

Hiroshige Tateuchi, PhD

Assistant Professor, Human Health Sciences, Graduate School of Medicine, Kyoto University

53 Kawara-cho, Shogoin, Sakyo-ku, Kyoto 606-8507, Japan

Tel: +81-75-751-3964

Fax: +81-75-751-3909

E-mail: tateuchi@hs.med.kyoto-u.ac.jp 


\begin{abstract}
Prone hip extension has been used as a self-perturbation task to test the stability of the lumbopelvic region. However, the relationship between recruitment patterns in the hip and trunk muscles and lumbopelvic kinematics remains unknown. The present study aimed to examine if the balance of hip and trunk muscle activities are related to pelvic motion and low back muscle activity during prone hip extension. Sixteen healthy participants performed prone hip extension from $30^{\circ}$ of hip flexion to $10^{\circ}$ of hip extension. Surface electromyography (of the gluteus maximus, semitendinosus, rectus femoris, tensor fasciae latae, multifidus, and erector spinae) and pelvic kinematic measurements were collected. Results showed that increased activity of the hip flexor (tensor fasciae latae) relative to that of hip extensors (gluteus maximus and semitendinosus) was significantly associated with increased anterior pelvic tilt during hip extension $(r=0.52)$. Increased anterior pelvic tilt was also significantly related to the delayed onset timing of the contralateral and ipsilateral multifidus $(r=0.57, r=0.53)$ and contralateral erector spinae $(r=0.63)$. Additionally, the decrease of the gluteus maximus activity relative to the semitendinosus was significantly related to increased muscle activity of the ipsilateral erector spinae $(\mathrm{r}=-0.57)$. These results indicate that imbalance between the agonist and antagonist hip muscles and delayed trunk muscle onset would increase motion in the lumbopelvic region.
\end{abstract}




\section{Introduction}

Active prone hip extension is often used as an exercise in physical therapy for the patients with hip or trunk dysfunction. This task also has been used as a self-perturbation task to test the stability of the lumbopelvic region (Janda, 1996; Sahrmann, 2002). Clinically, in patients with lumbopelvic dysfunction, the lumbopelvic region is often observed to extend or rotate excessively during prone hip extension (Sahrmann, 2002).

Previous studies have analyzed muscle activation patterns with respect to muscle firing order during active prone hip extension. Vogt and Banzer (1997) studied the sequential activation of lumbar and hip muscles in active prone hip extension. They found that there is a consistent muscle firing order of the ipsilateral lumbar erector spinae, semitendinosus, contralateral lumbar erector spinae, tensor fasciae latae, and gluteus maximus. Sakamoto et al. (2009) also reported the muscle activation order of the semitendinosus, ipsilateral and contralateral erector spinae, and gluteus maximus muscles in prone hip extension with knee flexion, knee extension, and hip lateral rotation and knee flexion. However, other studies indicated that there are no consistent recruitment patterns for prone hip extension among erector spinae, hamstrings, and gluteus maximus (Lehman et al. 2004, Pierce \& Lee 1990). Moreover, a report by Guimarães et al. (2010) has cast doubt over the possibility that patients with low back pain and the healthy individuals can be distinguished only by analyzing the muscle firing order. A previous study suggested that it is necessary to evaluate the movement patterns in addition to the muscle activation patterns during the active prone hip extension in order to discriminate between patients with low back pain and healthy individuals (Guimarães et al., 2010).

The factors affecting the lumbopelvic kinematics and activity of the low back muscles during hip extension could include muscle activity balance in the hip-joint muscles (balance between agonist and antagonist muscles as well as balance among the synergistic muscles) and muscle activity balance between the hip and trunk muscles (balance between the prime mover and lumbopelvic stabilizer). It is theoretically possible that altered balance of muscle activation amplitudes and muscle activation timing leads to altered movement patterns, favoring the occurrence of anterior pelvic tilt and excessive lumbar extension. However, no studies have examined the relationship between the balance in hip and trunk muscle activity and kinematic or muscle activity in the lumbopelvic region.

The purpose of this study was to examine if the balance of hip and trunk muscle activities are related to pelvic motion and low back muscle activity during prone hip extension. By examining these relationships, we will gain insights into the potential cause of lumbopelvic pathokinematics during prone hip extension.

\section{Methods}




\subsection{Participants}

Sixteen healthy subjects (10 men and 6 women) participated in the study. Their mean age was $24.3 \pm 5.2$ (mean $\pm \mathrm{SD}$ ) years, their mean body weight was $59.0 \pm 8.0 \mathrm{~kg}$, and their mean height was $165.7 \pm 7.9 \mathrm{~cm}$.

Subjects were excluded from the study if they had musculoskeletal conditions, or if they had been diagnosed with neurological disorders or cardiovascular disease that would limit their function. Subjects who had a hip extension angle less than $10^{\circ}$ were also excluded from the study. All of the subjects provided informed consent, and the protocol was approved by the Ethics Committee of the Kyoto University Graduate School and Faculty of Medicine.

\subsection{Experimental procedure}

The subjects were asked to lie on a table in the prone position with the right hip hanging over the edge of the table which was tilted down to $30^{\circ}$. Each subject was instructed to perform active hip extension from $30^{\circ}$ of flexion to $10^{\circ}$ of extension while keeping the knee extended. For each subject, the hip extension angle was defined by placement of a thin rope (Fig. 1). Fixation devices were not applied to the pelvis and trunk. The subjects raised the leg for $1 \mathrm{~s}$ after an LED indicator signal placed in front of the subjects was turned on. The hip was held in the extended position for at least 3 s. Prone hip extension was performed for 5 consecutive repetitions.

\subsection{Electromyography recording and data analysis}

After the electrode sites were shaved and cleaned with scrubbing gel and alcohol, disposable pre-gelled electromyography (EMG) $\mathrm{Ag}-\mathrm{AgCl}$ electrodes (Blue sensor; Medicotest Inc., Olstykke, Denmark) with a 2-cm center-to-center inter-electrode distance were applied over the following 8 muscles according to the SENIAM recommendations (SENIAM Web site): the bilateral lumbar erector spinae (ES: at a 2- finger-width distance lateral from the spinous process of L1), bilateral lumbar multifidus (MF: at the level of the L5 spinous process on a line extending from the posterior superior iliac spine to the interspace between L1 and L2), right gluteus maximus (Gmax: 50\% on the line extending between the sacrum and greater trochanter), right semitendinosus (ST: $50 \%$ on the line extending between the ischial tuberosity and medial epicondyle), right rectus femoris (RF: $50 \%$ on the line extending from the anterior superior iliac spine to the superior part of the patella), and right tensor fasciae latae (TFL: on the line extending from the anterior superior iliac spine to the lateral femoral condyle in the proximal 1/6). All electrode placements were confirmed through palpation 
and manual resistance. Raw EMG signals processed using an $8^{\text {th }}$-order Butterworth filter with a bandpass range of 10-500 Hz (CMR > 100dB) were amplified and collected with a sampling rate of 1,000 Hz using a 12-bit A/D converter with a $\pm 5-\mathrm{V}$ range (Telemyo 2400T V2; Noraxon USA Inc., Scottsdale, AZ). Manual resistance was applied to obtain maximal voluntary isometric contractions (MVICs) in the following positions: prone trunk extension for the trunk extensors, prone hip extension with knee flexion for the gluteus maximus, prone knee flexion for the semitendinosus, sitting knee extension for the rectus femoris, and sidelying hip abduction for the tensor fasciae latae. Subjects were instructed to generate muscle contraction force against the resistance, while the EMG signals were recorded during a stable $3 \mathrm{~s}$ as MVICs for each muscle.

The root-mean-squares (RMSs) of the raw data were determined, and 3-s MVICs were calculated for each muscle. For each individual muscle, the average RMS EMG amplitude was determined over the 3-s period, while the leg was maintained in the hip-extended position. The average RMS EMG amplitude of the each muscle was normalized to each of the MVICs. According to previous studies, a positive EMG signal was designated >5\% MVICs (Potvin and O'Brien, 1998; Ricamato and Dhaher, 2004; Zhang et al., 2009). Furthermore, after normalization, we calculated $(\mathrm{RF} \times 2) /(\mathrm{Gmax}+\mathrm{ST})$, $(\mathrm{TFL} \times 2) /(\mathrm{Gmax}+\mathrm{ST})$, and Gmax/ST to index the balance of hip muscle activity, and (contralateral $\mathrm{MF} \times 2) /(\mathrm{Gmax}+\mathrm{ST}),($ ipsilateral $\mathrm{MF} \times 2) /(\mathrm{Gmax}+\mathrm{ST}),($ contralateral $\mathrm{ES} \times 2) /(\mathrm{Gmax}+\mathrm{ST})$, and (ipsilateral $\mathrm{ES} \times 2) /(\mathrm{Gmax}+\mathrm{ST})$ to index the balance of hip and trunk muscle activity.

The onset of the muscle activity was determined using the cumulative sum (CUSUM) methods (Ando et al., 2009; Brodin et al., 1993). First, we rectified EMG from $500 \mathrm{~ms}$ before the LED signal to 1,000 ms after the LED signal. Second, the background EMG over $500 \mathrm{~ms}$ before the LED signal were averaged. The mean background EMG was subtracted from the rectified EMG. The rectified EMG was summed up over 1,000 ms after the LED signal, and the resulting value was defined as $100 \%$. The EMG onset was defined as the point at which the cumulative sum of the rectified EMG reached a threshold of 5\%. When we judged that the EMG onset was not appropriate by visual inspection, we changed the threshold with a step of $0.1 \%$. the EMG onset time was determined by a single blinded investigator. In order to investigate the temporal firing pattern among the hip and trunk muscles, the relative difference of the onset time between each muscle and the prime mover (the semitendinosus) was calculated (Chance-Larsen et al., 2010; Lehman et al., 2004). A positive value indicates that the semitendinosus muscle was getting activated earlier.

For the normalized RMS EMG amplitude and relative time difference of each muscle, the averages of the values obtained in the 5 repetitions were determined for subsequent analysis.

\subsection{Kinematics measurements}

Body kinematics data were recorded using a 6-camera Vicon motion system (Vicon Nexus; Vicon Motion Systems Ltd., Oxford, England) at a sampling rate of $200 \mathrm{~Hz}$. The subjects were clothed in 
close fitting shorts and T-shirts, and reflective markers were attached to the body of each subject. Seven reflective markers were placed as follows; bilaterally on the skin overlying the posterior superior iliac spine and the top of the iliac crest, and unilaterally over the greater trochanter, lateral epicondyle of the femur and lateral malleolus of the right leg (Fig. 2). All data were low-pass filtered using a Woltring filter with a cut-off frequency of $6 \mathrm{~Hz}$. A trigger mechanism was used to synchronize the EMG measurements and the motion-capture system data.

Three-dimensional angular displacements of the pelvis were calculated across time with reference to a standard according to the guidelines of the International Society of Biomechanics (Wu et al., 2002). The origin of the pelvic segment coincided with the right iliac crest. The Z-axis of the pelvis was defined as the line parallel to a line connecting the markers on the right and left iliac crest. The $\mathrm{X}$-axis was defined as the line parallel to a line lying in the plane defined by the 2 markers on the right and left iliac crest and the midpoint of the 2 markers on the posterior superior iliac spine, orthogonal to the Z-axis, and pointing anteriorly. The Y-axis was defined as the line perpendicular to both the $\mathrm{X}$ - and Z-axes, pointing cranially. The tilt about the Z-axis, oblique about the $\mathrm{X}$-axis, and rotation about the Y-axis of the pelvis were calculated (Fig. 2).

The onset of the pelvic motion was defined as the point at which the angular velocity of the anterior pelvic tilt exceeds $5 \%$ of the maximal angular velocity. The initiation of the lower leg motion was defined as the point at which the upward velocity of the marker on the knee (lateral femoral epicondyle) exceeded $5 \%$ of the maximal velocity. The termination of movement of the pelvis and knee marker was defined as the point at which the velocity was less than $5 \%$ of the maximum velocity (Sholtes et al., 2009).

The dependent variables were the three-dimensional angles of the pelvis and time of the initiation of the pelvic motion. Pelvic angles were calculated as the changes from the angles in the initial prone position. The time of the initiation of the pelvis was normalized to the limb movement time by dividing the start-time difference by the time required to complete the limb movement (Sholtes et al., 2009). The average values of the 5 repetitions were used for the analysis.

\subsection{Statistical analysis}

The Statistical Package for Social Sciences 17.0 (SPSS Inc.) was used for statistical analyses. Since the data that did not represent a normal distribution in Shapiro-Wilk tests were included in the determination of \%MVICs and muscle onset time, we applied non-parametric statistical methods. Relationships of the muscle activity balance in the hip-joint muscles and balance of hip and trunk muscles with pelvic motion and trunk muscle activities were examined using Spearman's rank correlation coefficients. The muscle firing order was evaluated using the Wilcoxon signed-rank test with Holm's correction for multiple comparisons. Relationships between onset timing of the activity of each muscle of trunk and hip muscles relative to that of the semitendinosus muscle and pelvic 
motion were also examined using Spearman's rank correlation coefficients. P value of $<0.05$ were considered significant.

\section{Results}

Mean \pm SD values of the activity of each of the muscles and pelvic motion during prone hip extension are shown in Table 1. The mean value of the rectus femoris muscle activity was found to be less than 5\% MVICs. Therefore, the rectus femoris muscle was excluded from the subsequent analysis.

3.1. Relationship of balance of hip and trunk muscle activities with pelvic motion and trunk muscle activity

Increased $(\mathrm{TFL} \times 2) /(\mathrm{Gmax}+\mathrm{ST})$ was found to be significantly associated with the anterior pelvic tilt ( $\mathrm{r}=0.52, \mathrm{p}=0.041$, Table 2, Fig.4 (a)). Furthermore, the decreased Gmax/ST was found to be significantly related to the increased EMG signal amplitude of the ipsilateral ES $(r=-0.57, p=$ 0.021 , Table 2).

\subsection{Muscle firing order during prone hip extension}

As shown in Fig. 3, the mean onset time of all hip and trunk muscles except Gmax was earlier than the onset of leg movement. The onset timing of Gmax was delayed significantly relative to the onset timing of the contralateral MF $(\mathrm{p}=0.027)$, ipsilateral MF $(\mathrm{p}=0.008)$, contralateral $\mathrm{ES}(\mathrm{p}=$ $0.038)$, ipsilateral ES $(\mathrm{p}=0.035)$, and the ST $(\mathrm{p}=0.009)$. Furthermore, the ipsilateral MF was activated earlier than the contralateral ES $(\mathrm{p}=0.018)$. There was no consistent recruitment pattern observed among the trunk muscles and ST.

\subsection{Relationship between onset timing of each muscle activity and pelvic motion}

There was no significant correlation between the onset timing of the activity of each muscle and the onset timing of the pelvic motion (Table 3). However, a delay of the onset timing of the contralateral MF ( $r=0.57, p=0.020$, Fig. $4(b))$, the ipsilateral MF $(r=0.53, p=0.037$, Fig. 4 (c)), and the contralateral ES $(r=0.63, p=0.009$, Fig. $4(d))$ relative to ST firing were significantly associated with an increase of the anterior pelvic tilt (Table 3). 


\section{Discussion}

The primary findings of the current study were that excessive activity of the hip flexor (tensor fasciae latae) relative to the hip extensors (gluteus maximus and semitendinosus) and delay of firing of the bilateral multifidus and contralateral erector spinae were related to increased anterior tilt of the pelvis during prone hip extension. Although excessive extension and rotation of the lumbopelvic region is observed in patients with lumbopelvic dysfunction in the clinical setting (Sahrmann, 2002), the anterior pelvic tilt would be more susceptible to the altered muscle activation patterns of the hip and trunk muscles. Furthermore, a decrease of the gluteus maximus activity relative to the semitendinosus muscle was found to be associated with excessive muscle activity of the ipsilateral erector spinae. Our results suggest that there is a need to take particular note of imbalance of the muscle activity in the hip-joint muscles and altered onset timing of the trunk muscles in evaluation of lumbopelvic pathokinematics.

Increased activity of the tensor fasciae latae relative to the hip extensors was identified as a factor related to the increased anterior tilt of the pelvis. Increased coactivation of antagonists results in an increase in compressive force acting on the joint (which contributes to the increase in joint stiffness) but a reduction in the net moment acting on the joint. (Klass et al., 2007; Pereira and Concalves, 2011; Zeni and Higginson, 2009). In prone hip extension, activation of the antagonistic tensor fascia latae would counteract the extension motion of the hip joint. The height of the extended femur was prescribed uniformly in the present study. Therefore, hip extension would be compensated by an excessive anterior tilt of the pelvis in case of high activity of the tensor fascia latae. Recently, it has been reported that an abdominal drawing-in maneuver, which induces activation of the transverse abdominis, is an effective method for decreasing the anterior pelvic tilt during prone hip extension (Oh et al., 2007). However, improvement of the hip muscle balance between agonist and antagonist (i.e., facilitation of the agonist muscles or inhibition of the antagonist muscles) may be considered for reduction of the anterior pelvic tilt.

Decreased Gmax/ST (i.e., the activity of the semitendinosus was relatively dominant) was found to be associated with increased activity of the ipsilateral erector spinae. Although the gluteus maximus and hamstring muscles are prime movers in the process of hip extension, these 2 muscles have different features with respect to alterations in the muscle moment arm according to the change in the hip flexion-extension angle (Németh and Ohlsén, 1985; Pohtilla, 1969). The moment arm of the gluteus maximus increases with increase in the hip extension angle. On the other hand, the semitendinosus showed a decrease in the moment arm with increasing hip extension (Németh and Ohlsén, 1985; Pohtilla, 1969). Based on these observations of the muscle moment arm, it appears that the gluteus maximus has a relative advantage for providing extension force production in the hip-extended position, although both muscles are shortened with hip extension. In previous research 
of the EMG/angle relationship, the muscle activity of the gluteus maximus at $0^{\circ}$ of hip flexion was found to be greater than that at the hip flexed position, while the hamstring muscles showed no significant differences with changes of the hip angle (Worrell et al., 2001). Decreased Gmax/ST might reduce the efficiency of force production of the hip extension in the hip-extended position, and as a result, the erector spinae may need to increase its activity to maintain the anterior pelvic tilt and spine extension.

Although several studies have investigated the order of muscle firing in the active prone hip extension, there is no consistent order pattern even in healthy individuals. However, delayed onset of the gluteus maximus has been identified consistently. Our result is in agreement with previous studies in terms of the delayed onset of the gluteus maximus relative to the semitendinosus and erector spinae (Lehman et al, 2004; Sakamoto et al, 2009; Vogt and Banzer, 1997).

Research into the muscle firing order in prone hip extension has been mainly focused on the gluteus maximus, semitendinosus, and erector spinae. However, no studies have been conducted on the lumbopelvic stabilizer muscles such as the multifidus. The feedforward window has been defined by activation of the anticipatory muscles $100 \mathrm{~ms}$ before EMG onset of the prime mover to $50 \mathrm{~ms}$ afterward (Aruin and Latash, 1995; Hodges and Richardson, 1997). Thus, in the current study, the action of the bilateral erector spinae and multifidus could be interpreted as feedforward activity at initiation of active prone hip extension. Furthermore, the present result shows that the onset of the ipsilateral multifidus occurred significantly earlier than that of the contralateral erector spinae. This indicates the importance of including the multifidus muscle in the analysis of the prone hip extension. Although previous research has identified feedforward activation of the multifidus in association with limb movements that challenge the stability of the spine (Hodges and Richardson, 1997), the multifidus is expected to be a critical muscle for active prone hip extension since the multifidus provides an essential source of extension, rotation torque, and stability to the base of the spine (Kendall et al, 2005; Neumann, 2002).

From the viewpoint of the relationship between the muscle onset time and motion pattern, we found that the onset delay of the bilateral multifidus and contralateral erector spinae relative to that of the semitendinosus were associated with increased anterior pelvic tilt. The multifidus and erector spinae have an extension function on the lumbar spine, while the multifidus in particular has a stabilizing effect on the lumbar spine. Wilke et al. (1995) demonstrated that simulated muscle force of the entire multifidus muscle group reduced the range of motion not only in the flexion and rotation but also in the extension of the lumbar spine. Since prone hip extension is a self-perturbation task for testing the stability of the lumbopelvic region, onset delay of a lumbopelvic stabilizer such as the multifidus might cause instability of the lumbopelvic region during leg motion, eventually increasing the extent of anterior pelvic tilt in the hip-extended position. A previous study has shown that there is a delay in the onset of activation of the multifidus as well as for the internal oblique muscle at the point of initiation of weight transfer during hip flexion while standing on the symptomatic side in patients with sacroiliac joint pain as compared to control subjects and to the asymptomatic side of the 
patients (Hungerford et al., 2003). Moreover, Silfies et al. (2009) have reported that later onset of the activity of the lumbar multifidus and erector spinae occur during a rapid shoulder flexion task in patients with low back pain and segmental instability relative to control subjects and low back pain patients without instability. Although the relationship between the delayed onset of muscle activity and lumbopelvic kinematic changes have not been analyzed in patients with lumbopelvic dysfunction, our results suggest that delay of trunk muscle activation may be a potential source of change in motion of the lumbopelvic region. Further research would be necessary to completely test this hypothesis.

Regarding the limitations of this study, the first limitation is that the EMG of other hip extensor muscles (e.g., posterior fibers of the adductor magnus) and other hip flexor muscles that function as antagonists (e.g., the iliopsoas, adductor longus, or sartorius) were not measured. Furthermore, we recorded measurements of the superficial multifidus using surface EMG, although the superficial multifidus and deep multifidus are considered to be differentially active. Specifically, it was reported that, unlike superficial multifidus, deep multifidus was activated in a non-direction-specific feedforward manner in association with rapid arm movement, and muscle activity during predictable loading were less in deep multifidus rather than in superficial multifidus in patients with low back pain (MacDonald et al., 2006; Moseley et al., 2002; MacDonald et al., 2010). However, the gluteus maximus and hamstrings are prime movers in hip extension while the extension force produced by the adductor magnus is decreased with hip extension since the moment arm is closer to $0 \mathrm{~mm}$ (Németh and Ohlsén, 1985). Additionally, with regard to the multifidus, onset delay during a self-perturbation task and less activity as well as deep multifidus activity during unpredictable loading were identified even in the superficial multifidus in individuals with recurrent low back pain (MacDonald et al., 2010; Silfies et al., 2009). Therefore, we considered the results to be valid for this study to examine the essential relationship of muscle activity balance and relative timing of the hip and trunk muscle with lumbopelvic kinematics and muscle activity. The second limitation is that the intersegmental motion of the lumbar spine was not measured. Although three-dimensional rotation of the pelvis appears to be directly linked to the movement of the lumbar spine (Levine and Whittle, 1996), more detailed research would be needed for confirmation. Finally, prone hip extension and standing tasks or walking differ in their gravity direction. Thus, it is unknown whether the same phenomenon observed in this study also occurs in tasks performed in the upright standing position.

In conclusion, increased activity of the hip flexor (tensor fasciae latae) relative to the hip extensors (the gluteus maximus and semitendinosus) and delayed onset of firing of the bilateral multifidus and contralateral erector spinae were associated with an increased anterior pelvic tilt during prone hip extension. Furthermore, a decrease in the activity of the gluteus maximus relative to the activity of the semitendinosus was related to increased muscle activity of the ipsilateral erector spinae. We propose that alterations in the balance of muscle activity in hip-joint muscles and relative timing of the activity of the hip and trunk muscles may lead to increased motion in the lumbopelvic region. 


\section{Acknowledgements}

The authors wish to thank Masahiro Kokubu, Shingo Takashima, and Yusuke Okita for their technical assistance with data analyses.

\section{References}

Ando S, Yamada Y, Tanaka T, Oda S, Kokubu M. Reaction time to peripheral visual stimuli during exercise under normoxia and hyperoxia. Eur J Appl Physiol 2009;106:61-9.

Aruin AS, Latash ML. Directional specificity of postural muscles in feed-forward postural reactions during fast voluntary arm movements. Exp Brain Res 1995;103(2):323-32.

Brodin P, Miles TS, Türker KS. Simplereaction-time responses to mechanical and electrical stimuli in human masseter muscle. Arch Oral Biol 1993;38(3):221-6.

Chance-Larsen K, Littlewood C, Garth A. Prone hip extension with lower abdominal hollowing improves the relative timing of gluteus maximus activation in relation to biceps femoris. Man Ther 2010;15(1):61-5.

Guimarães CQ, Sakamoto AC, Laurentino GE, Teixeira-Salmela LF. Electromyographic activity during active prone hip extension did not discriminate individuals with and without low back pain. Rev Bras Fisioter 2010;14(4):351-7.

Hodges PW, Richardson CA. Contraction of the abdominal muscles associated with movement of the lower limb. Phys Ther 1997;77(2):132-42; discussion 142-4.

Hungerford B, Gilleard W, Hodges P. Evidence of altered lumbopelvic muscle recruitment in the presence of sacroiliac joint pain. Spine (Phila Pa 1976) 2003;28(14):1593-600.

Janda V. Evaluation of muscular imbalance. In: Liebenson C, editor. Rehabilitation of the spine: a practitioner's manual. 1st ed. Baltimore: Lippincott Williams \& Wilkins, 1996: 97-112.

Kendall FP, McCreary EK, Provance PG, Rodgers MM, Romani WA. Muscles: Testing and function with posture and pain. 5th ed. Baltimore: Lippincott Williams \& Wilkins, 2005.

Klass M, Baudry S, Duchateau J. Voluntary activation during maximal contraction with advancing age: a brief review. Eur J Appl Physiol 2007;100(5):543-51.

Lehman GJ, Lennon D, Tresidder B, Rayfield B, Poschar M. Muscle recruitment patterns during the prone leg extension. BMC Musculoskelet Disord 2004;5:3.

Levine D, Whittle MW. The effects of pelvic movement on lumbar lordosis in the standing position. J Orthop Sports Phys Ther 1996;24(3):130-5.

MacDonald DA, Moseley GL, Hodges PW. The lumbar multifidus: does the evidence support clinical beliefs? Man Ther 2006;11(4):254-63. 
MacDonald D, Moseley GL, Hodges PW. People with recurrent low back pain respond differently to trunk loading despite remission from symptoms. Spine (Phila Pa 1976) 2010;35(7):818-24.

Moseley GL, Hodges PW, Gandevia SC. Deep and superficial fibers of the lumbar multifidus muscle are differentially active during voluntary arm movements. Spine (Phila Pa 1976) 2002;27(2):E29-36.

Nemeth G, Ohlsen H. In vivo moment arm lengths for hip extensor muscles at different angles of hip flexion. J Biomech 1985;18(2):129-40.

Neumann DA. Kinesiology of the musculoskeletal system: Foundations for physical rehabilitation. Mosby. Missouri, 2002

Oh JS, Cynn HS, Won JH, Kwon OY, Yi CH. Effects of performing an abdominal drawing-in maneuver during prone hip extension exercises on hip and back extensor muscle activity and amount of anterior pelvic tilt. J Orthop Sports Phys Ther 2007;37(6):320-4.

Pereira MP, Concalves M. Muscular coactivation (CA) around the knee reduces power production in elderly women. Arch Gerontol Geriatr 2011;52(3):317-21.

Pierce MN, Lee WA. Muscle firing order during active prone hip extension. J Orthop Sports Phys Ther 1990;12(1):2-9.

Pohtilla JF. Kinesiology of hip extension at selected angles of pelvifemoral extension. Arch Phys Med Rehabil 1969;50(5):241-50.

Potvin JR, O'Brien PR. Trunk muscle co-contraction increases during fatiguing, isometric, lateral bend exertions. Possible implications for spine stability. Spine (Phila Pa 1976) 1998;23(7):774-80.

Ricamato AL, Dhaher YY. Electrical cortical activity associated with joint torque direction in the human arm. J Clin Neurophysiol 2004;21(3):192-208.

Sahrmann SA. Diagnosis and treatment of movement impairment syndromes. 1st ed. Missouri: Mosby, 2002.

Sakamoto AC, Teixeira-Salmela LF, de Paula-Goulart FR, de Morais Faria CD, Guimaraes CQ. Muscular activation patterns during active prone hip extension exercises. J Electromyogr Kinesiol 2009;19(1):105-12.

Scholtes SA, Gombatto SP, Van Dillen LR. Differences in lumbopelvic motion between people with and people without low back pain during two lower limb movement tests. Clin Biomech (Bristol, Avon) 2009;24(1):7-12.

SENIAM Web site. Available at: http://www.seniam.org. Accessed: December 1, 2009.

Silfies SP, Mehta R, Smith SS, Karduna AR. Differences in feedforward trunk muscle activity in subgroups of patients with mechanical low back pain. Arch Phys Med Rehabil 2009;90(7):1159-69.

Vogt L, Banzer W. Dynamic testing of the motor stereotype in prone hip extension from neutral position. Clin Biomech (Bristol, Avon) 1997;12(2):122-127.

Wilke HJ, Wolf S, Claes LE, Arand M, Wiesend A. Stability increase of the lumbar spine with 
different muscle groups. A Biomechanical in vitro study. Spine (Phila Pa 1976) 1995;20(2):192-8.

Worrell TW, Karst G, Adamczyk D, Moore R, Stanley C, Steimel B, Steimel S. Influence of joint position on electromyographic and torque generation during maximal voluntary isometric contractions of the hamstrings and gluteus maximus muscles. J Orthop Sports Phys Ther 2001;31(12):730-40.

Wu G, Siegler S, Allard P, Kirtley C, Leardini A, Rosenbaum D, Whittle M, D’Lima DD, Cristofolini L, Witte H, Schmid O, Stokes I. ISB recommendation on definitions of joint coordinate system of various joints for the reporting of human joint motion--part I: ankle, hip, and spine. International Society of Biomechanics. J Biomech 2002;35(4):543-8.

Zeni JA Jr, Higginson JS. Dynamic knee joint stiffness in subjects with a progressive increase in severity of knee osteoarthritis. Clin Biomech 2009;24(4):366-71.

Zhang Q, Jonasson C, Styf J. Simultaneous intramuscular pressure and surface electromyography measurement in diagnosing the chronic compartment syndrome. Scand J Med Sci Sports 2009:22:1-6. 
Table 1: Descriptive data of hip and trunk muscle activity and pelvic motion during prone hip extension

\begin{tabular}{lcc}
\hline & Mean & SD \\
\hline EMG (\%MVICs) & & \\
Gluteus maximus & 10.88 & 3.33 \\
Semitendinosus & 7.63 & 2.79 \\
Tensor fasciae latae & 5.94 & 4.80 \\
Rectus femoris & 2.68 & 1.97 \\
Contralat. Multifidus & 11.86 & 4.03 \\
Ipsilat. Multifidus & 14.46 & 6.88 \\
Contralat. Erector spinae & 12.36 & 3.05 \\
Ipsilat. Erector spinae & 10.79 & 3.32 \\
Pelvic motion ( $\left.{ }^{\circ}\right)$ & & \\
Tilt (+: anterior tilt) & 6.71 & 4.38 \\
Oblique (+: rise of right iliac crest) & 0.39 & 0.81 \\
Rotation & 0.68 & 4.01 \\
$\quad(+:$ backward rotation of right iliac crest) & & \\
\hline
\end{tabular}


Table 2: Spearman's correlation among balance of hip and trunk muscle activity, pelvic motion, and trunk muscle activity

\begin{tabular}{|c|c|c|c|c|c|c|c|}
\hline & \multicolumn{3}{|c|}{ Pelvic motion } & \multicolumn{4}{|l|}{ Trunk muscle } \\
\hline & Tilt & Oblique & Rotation & Contralat. MF & Ipsilat. MF & Contralat. ES & Ipsilat. ES \\
\hline $\begin{array}{l}(\mathrm{TFL} \times 2) / \\
\quad(\mathrm{Gmax}+\mathrm{ST})\end{array}$ & $0.52^{*}$ & 0.13 & 0.09 & 0.16 & -0.09 & 0.02 & 0.09 \\
\hline $\mathrm{Gmax} / \mathrm{ST}$ & 0.04 & -0.13 & 0.14 & -0.05 & -0.26 & -0.05 & $-0.57^{*}$ \\
\hline $\begin{array}{c}(\text { Contralat. MF } \times 2) / \\
(\text { Gmax }+ \text { ST })\end{array}$ & 0.25 & 0.37 & 0.27 & - & - & - & - \\
\hline $\begin{array}{l}(\text { Ipsilat. MF } \times 2) / \\
(\mathrm{Gmax}+\mathrm{ST})\end{array}$ & 0.18 & 0.18 & -0.18 & - & - & - & - \\
\hline $\begin{array}{c}(\text { Contralat. ES × 2)/ } \\
(\text { Gmax }+\mathrm{ST})\end{array}$ & 0.11 & 0.18 & 0.14 & - & - & - & - \\
\hline $\begin{array}{c}\text { (Ipsilat. ES × 2)/ } \\
(\mathrm{Gmax}+\mathrm{ST})\end{array}$ & 0.18 & 0.44 & 0.17 & - & - & - & - \\
\hline
\end{tabular}

\section{(Footnote for Table 2)}

Gmax $=$ Gluteus maximus, $\mathrm{ST}=$ Semitendinosus, $\mathrm{TFL}=$ Tensor fasciae latae, $\mathrm{MF}=$ Multifidus, and $\mathrm{ES}=$ Erector spinae.

$* P<0.05$ 
Table 3: Spearman's correlation between the onset time of each muscle (relative to the semitendinosus) and the onset and extent of pelvic motion

\begin{tabular}{lcccc}
\hline & $\begin{array}{c}\text { Onset of the } \\
\text { pelvic motion }\end{array}$ & Pelvic motion & & \\
& 0.22 & 0.27 & 0.22 & 0.01 \\
Gluteus maximus & 0.09 & $0.57^{*}$ & 0.24 & 0.03 \\
Contralat. Multifidus & 0.26 & $0.53^{*}$ & 0.31 & 0.03 \\
Ipsilat. Multifidus & 0.27 & $0.63^{* *}$ & 0.32 & 0.28 \\
Contralat. Erector spinae & 0.08 & 0.16 & 0.48 & 0.04 \\
Ipsilat. Erector spinae & & & & Rotation \\
\hline
\end{tabular}

(Footnote for Table 3)

*P<0.05, **P<0.01 


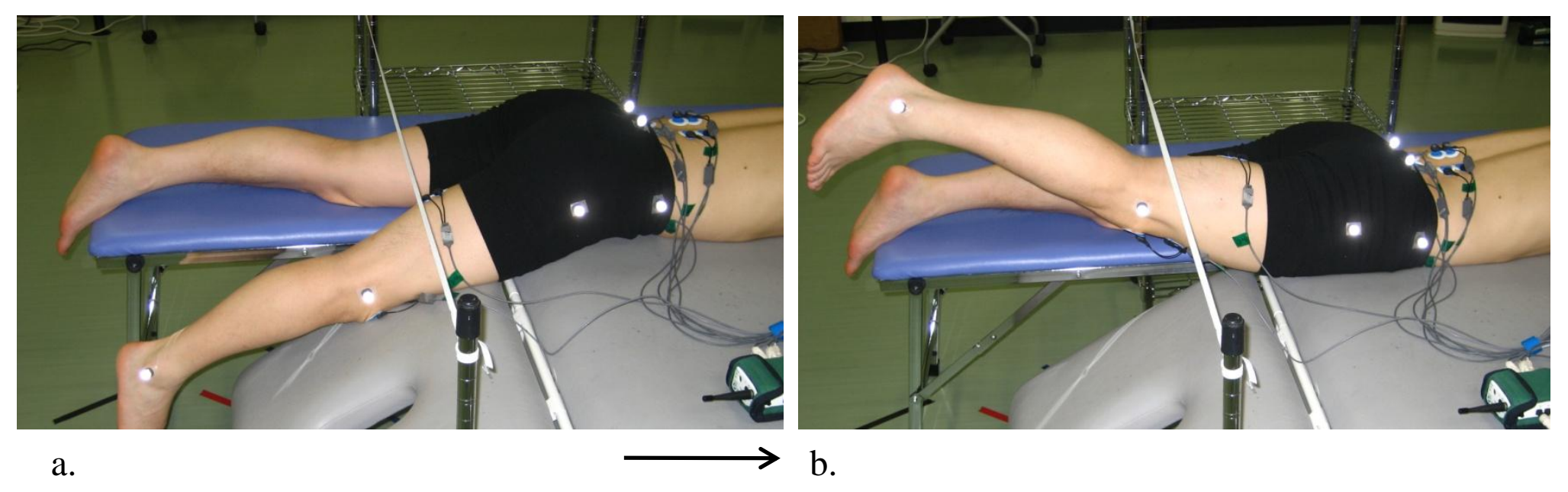

Fig. 1. 


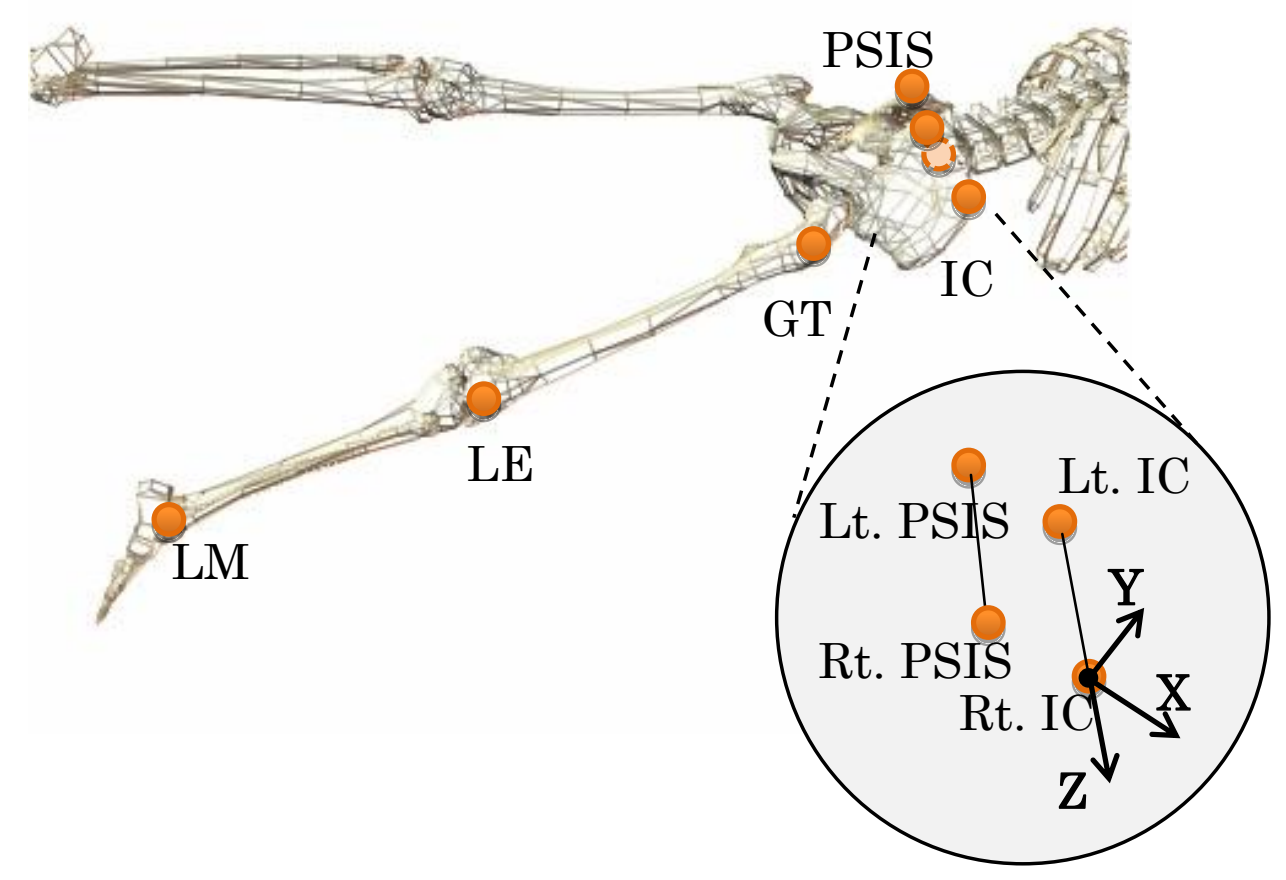

Fig. 2. 


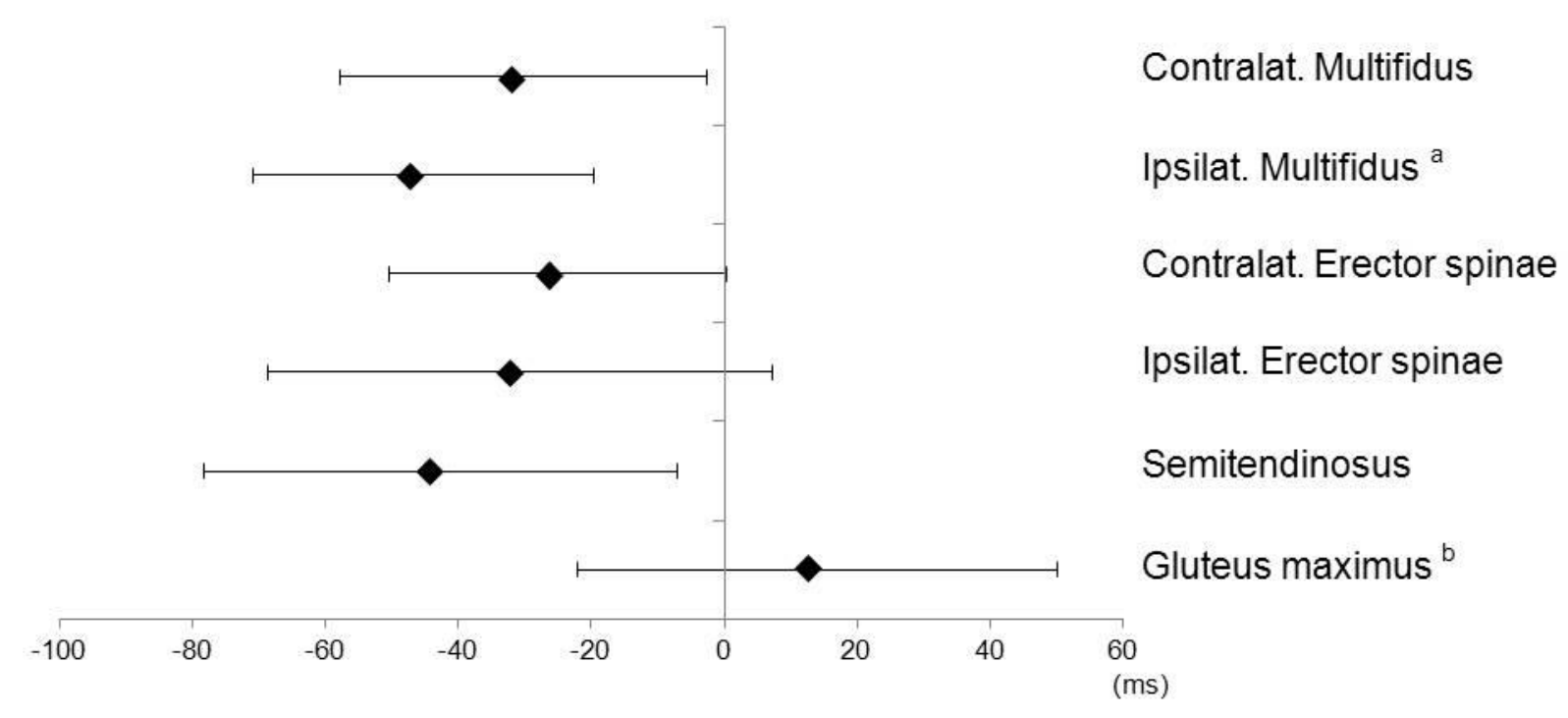

Fig. 3. 


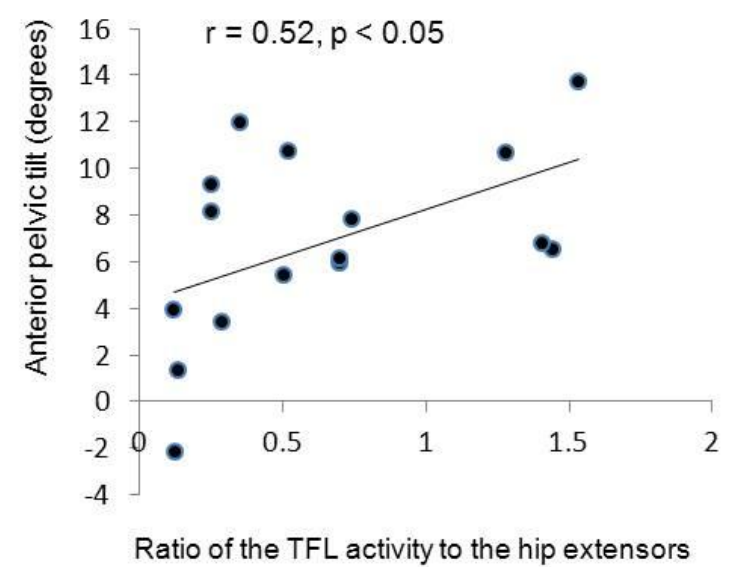

a.

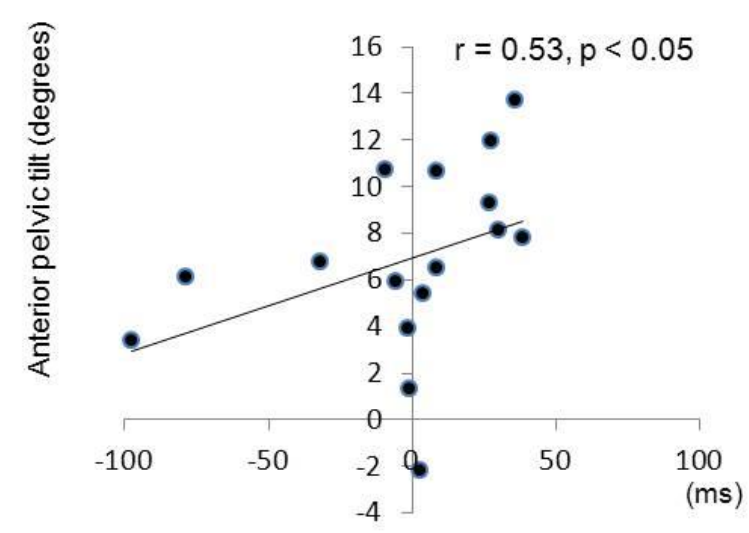

Onset time of the ipsilateral MF relative to the ST c.

Fig. 4.

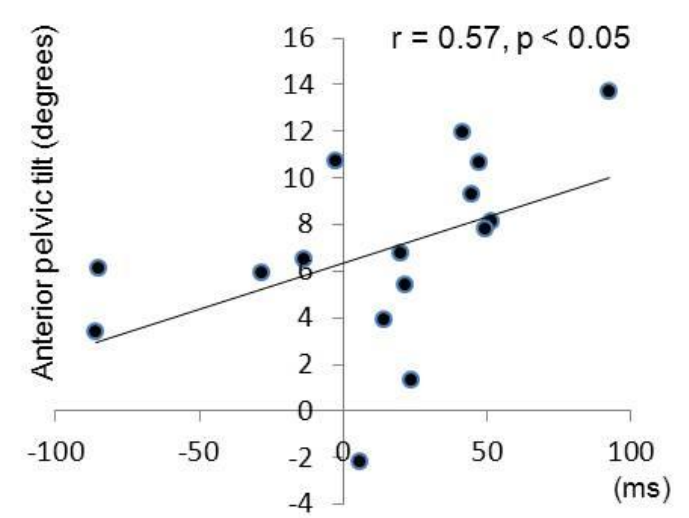

b.

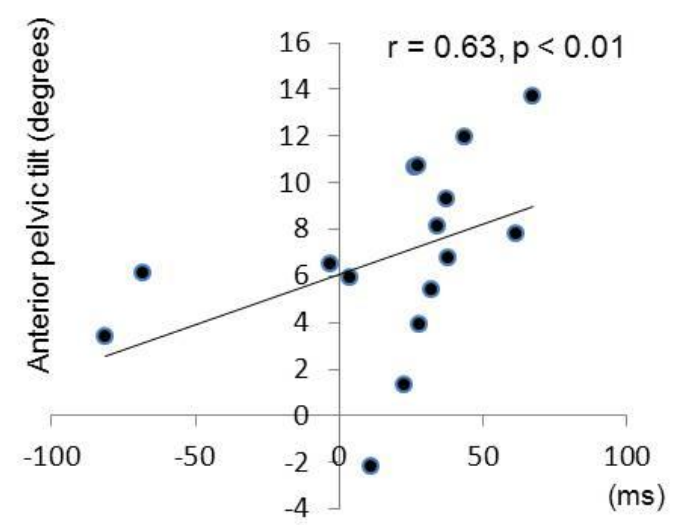

Onset time of the contralateral ES relative to the ST d. 


\section{Captions to figures}

Fig. 1. Active prone hip extension from the $30^{\circ}$ hip-flexed position (a) to the $10^{\circ}$ hip-extended position (b).

Fig. 2. Marker placements and definitions of the pelvic segment axes. PSIS = posterior superior iliac spine, IC = iliac crest, $\mathrm{GT}=$ greater trochanter, $\mathrm{LE}=$ lateral epicondyle of femur, and $\mathrm{LM}=$ lateral malleolus .

Fig. 3. Mean value and standard deviations are shown for onset times of the hip and trunk muscles. Time 0 represents the onset of leg motion. The ipsilateral multifidus was activated significantly earlier than the contralateral erector spinae (a). The gluteus maximus was activated significantly later than the semitendinosus and the trunk muscles (b).

Fig. 4. Relationship between anterior pelvic tilt and factors relevant to it. Anterior pelvic tilt and ratio of the TFL activity to the hip extensors (Gmax and ST) activity (a), anterior pelvic tilt and onset time of the contralateral MF relative to the ST (b), anterior pelvic tilt and onset time of the ipsilateral MF relative to the ST (c), and anterior pelvic tilt and onset time of the contralateral ES relative to the ST (d). Positive onset times indicate a later onset relative to ST in graphs $b, c$, and $d$. 


\section{Hiroshige Tateuchi}

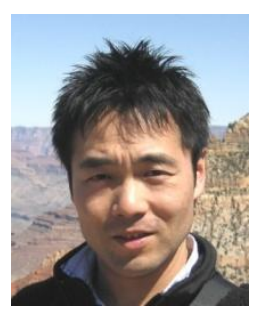

Hiroshige Tateuchi received his PhD from Kyoto University in 2011. Since 2006 he has been working as an assistant professor in the Department of Physical Therapy at the Graduate School of Medicine of Kyoto University. His main research interests are focused on EMG and biomechanical analysis of human movement and gait analysis in patients with musculoskeletal disorders.

\section{Masashi Taniguchi}

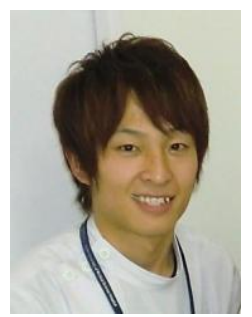

Masashi Taniguchi graduated from the Osaka Prefectural University in 2009 with a Bachelor of Physical Therapy. In 2011 he completed the Master of Human Health Sciences from Kyoto University. At present he works at the Division of Physical Therapy in the Shiga University of Medical Science Hospital as a physical therapist. His main interest is in clinical and biomechanical investigation on the human movement.

\section{Natsuko Mori}

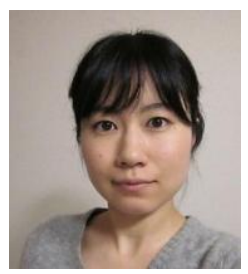

Natsuko Mori graduated with a Bachelor of Physical Therapy from the Hirosaki University in 2009. She completed the Master of Human Health Sciences from Kyoto University. She currently works at the Hirosaki Stroke and Rehabilitation Center as a physical therapist. Her primary research interests are in the area of physical therapy for urinary incontinence.

\section{Noriaki Ichihashi}

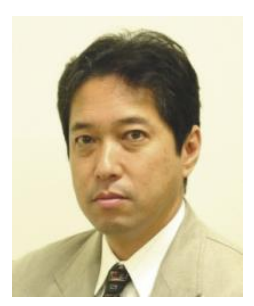

Noriaki Ichihashi is a professor in the Department of Physical Therapy at the Graduate School of Medicine of Kyoto University. He received his $\mathrm{PhD}$ from Fujita Health University in 2004. His field of research includes EMG analysis of muscle training; morphological analysis of muscle using ultrasoography; biomechanics of human movement; mechanism of kinematic chain; and fall in elderly people. 